\title{
SPECTRAL ENERGY DISTRIBUTION OF THE LIGHT EMITTED BY PLANTS AND ANIMALS
}

\author{
By W. W. Coblentz and C. W. Hughes
}

\begin{abstract}
The spectral energy determinations were made by a photographic method, by comparison with the spectral energy of a standard glowlamp.

The materials examined were a luminescent fungus (Agaricus melleus) found on decaying wood, a luminous crustacean (Cypridina), a firefly (Photinus pyralis) and two phosphorescent zinc sulphides.

The spectral energy distribution is as follows: In the luminous fungus it is rery wide and unsymmetrical, extending from 0.43 to $0.67 \mu$ with a strong maximum at $0.52 \mu$, and possibly a weak maximum at $0.58 \mu$; in the crustacean it extends from 0.41 to $0.62 \mu$ with a symmetrical distribution about the maximum at $0.48 \mu$; in the firefly the energy distribution is unsymmetrical about the maximum at $0.565 \mu$; in the zinc sulphide, $\mathrm{ZnS}$, the maximum is at $0.52 \mu$; and in the orange-colored zinc cadmium sulphide, $\mathrm{ZnCdS}$, there seem to be two maxima at $0.52 \mu$ and $0.61 \mu$, respectively.
\end{abstract}

\section{CONTENTS}

I. Introduction

II. Apparatus and procedure

III. Observations on luminous wood

1. Effect of sunlight and ultra-violet light

2. Observations on the isolated mycelium

3. The spectral energy curve of the luminous fungus

IV. Spectral energy distribution of the light emitted by cypridina

V. Spectral energy distribution of the light of the firefly

1. Comments on fireflies and glowworms in Sumatra.

VI. Spectral energy distribution of some phosphorescent zinc sulphides_.

\section{INTRODUCTION}

The physical characteristics and the chemical processes underlying bioluminescence are practically an unexplored subject.

The present paper gives the results of an investigation of the distribution of energy in the spectrum of the light emitted by plant and animal organisms. For completeness the spectral energy curves of several phosphorescent minerals are included in the work. 
In a previous paper ${ }^{1}$ it was shown that the light emitted by various species of fireflies differs greatly in spectral energy distribution. In an unforeseen and unexpected manner this information has proved useful in showing which is the light-producing and which is the light-giving substance (luciferin and luciferase) that is obtainable from the firefiy. By dialysis Harvey ${ }^{2}$ was able to show that the material obtained from the luminous organs of fireflies can be separated into two constituents which are essential in light production. These two constituents are (1) a heat-resistant, dialyzable substance called luciferin which takes up oxygen and oxidizes with light production in the presence of (2) a heat-sensitive, nondialyzable, enzymelike substance called tuciferase. The latter appears to be an organic catalyzer which accelerates the oxidation of the luciferin, the intensity of the luminescence being dependent upon the reaction velocity or rate of oxidation. By mixing the luciferin of one species of firefly with the luciferase of another species he was able to show that the light produced is characteristic of the animal supplying the luciferase.

In the animal kingdom there are many species which emit light; and more information seems to be at hand regarding the chemistry of light production in animals than in plants. In a recent survey of the subject by Harvey ${ }^{3}$ it was disclosed that there are at least 36 orders of animals, containing one or more forms known to produce light.

In the plant kingdom ${ }^{4}$ the only forms of life with an autogenous light production seem to be certain bacteria and fungi. Among the latter the light production is most easily observed in the vast numbers of threads, the mycelium, which grow in the decaying roots and stumps of trees.

For more than three centuries it has been known that this light production requires oxygen and that it is not a heat-producing combustion of the wood. The mycelium, lives for years and the light of a luminous form of fungus seems to continue while active metabolism is in progress.

Although the extent of the spectrum of a number of luminous organisms has been photographed, but little information is at hand concerning the distribution of the energy in the spectrum of the light emitted. In all cases thus far recorded the light emitted by the luminous organism appears to be only of wave lengths that con-

\footnotetext{
1 Coblentz, A Pbysical Study of the Firefly. Publ. No. 164, Carnegie Institution of Washington; 1912.

2 E. N. Harvey, The Nature of Animal Light, Lippincotts ; 1920.

s Harvey, The Nature of Animal Light; 1920.

"Mangold, Winterstcin's Handb. der Vergleich. Physiologie, 3; 1911. Dahlgren, "The production of light by animals," J. Franklin Inst., 180, pp. $515,711,1915 ; 181$, pp. 109 , 243, 377, 525, 659, 805, 1916.
} 
stitute what is known as the visible spectrum; but the spectral rango is much shorter than that to which the eye is sensitive. $\Lambda$ n interesting question in the physical production of light is why bioluminescent radiation (and to a great extent phosphorescence and fluorescence) should be of these short wave lengths.

In the previous investigation of the light emitted by the firefly it was shown conclusively that the color of the light is not a subjective visual phenomenon due to the Purkinje effect and to the low intensity of the light, but is owing to an actual difference in the spectral composition of the light emitted. The results of the present investigation are in agreement with the previous work.

The present investigation deals principally with a luminescent fungus (Agaricus melleus) found in decaying wood, which emits a faintly luminous greenish or yellowish-white light, popularly known as "fox fire"; also a luminescent crustacean (Cypridina). The latter consisted of a sealed glass tube of the dry, powdered organisms (presented by Prof. E. N. Harvey) which emit an intense bluish light when moistened with water. Although this material had been collected and sealed up over 6 years ago, it seemed to be just as photogeneous as when first received.

\section{APPARATUS AND PROCEDURE}

The spectrometer used in photographing the spectrum was provided with lenses $5 \mathrm{~cm}$ in diameter and $40 \mathrm{~cm}$ focal length and a flint-glass prism. The spectrometer was inclosed in a box to exclude stray light. This was placed in a dark room. The entrance slit, $5 \mathrm{~mm}$ high, was covered with white cardboard to enable the operator to locate the slit by means of the light emitted by the firefly and by the luminescent wood.

As in previous investigations the firefly was held so that the luminous segments covered the slit. When photographing the light emitted by the fungus, the piece of wood was placed upon a suitable support in front of the slit, and for the exposure during the night it was surrounded with moist cotton. The sample was then covered with a dark cloth to eliminate stray light.

Owing to the low intrinsic brightness, exposures of 24 to 71 hours, with frequent renewals of freshly glowing material, were required in order to obtain a good spectrum of the luminescent fungus. The exposures of the wood were continuous, day and night, the last sample being mounted at $9 \mathrm{p}$. m. and removed the next morning. From the behavior of the samples during the daytime it was estimated that the last sample mounted in the evening would continue to glow brightly for 3 to 4 hours, while some samples were found to be still glowing faintly the following morning. 
The spectrum was photographed on Wratten and Wainwright "panchromatic" plates as in the previous work. ${ }^{5}$ In order to obtain the energy involved in producing the spectrum of the light emitted by the firefly and by the fungus it was necessary to determine the "characteristic curve" of the photographic plate. This was accomplished by making a series of exposures, of known relationship, to a standard source (carbon glowlamp) of known spectral energy distribution. The exposures, which were for $2,4,6,8,10,12,20,30$, and 60 seconds' duration, were timed so as to cover the range of densities obtained in the unknown source; for example, the spectrum of the light of the firefly. In some cases all the exposures were obtained on the same plate; but for the luminous wood the slit had become soiled, causing a streaked comparison spectrum of the glowlamp. It was, therefore, necessary to use an additional series of exposures of the glowlamp on a separate plate.

An attempt was made to develop all the plates under the same conditions, in complete darkness. A fresh supply of developer was taken from a stock solution for each plate. The plates were developed for 4 minutes, the temperature of the developer at the start being $17^{\circ} \mathrm{C}$.

The spectral energy distribution of the standard glowlamp was determined anew and found in excellent agreement with the measurements made more than a dozen years ago.

The "densities" or blackening of the photographic plate were determined by means of a Martens polarization photometer, as described and illustrated in the previous publication. ${ }^{6}$ For this purpose the photometer openings were covered with black paper, with slits $1 \mathrm{~mm}$ wide and $1.5 \mathrm{~mm}$ high. This was mounted upon a large comparator obtained from the spectroscopy section of this bureau.

The spectral opacities or densities are illustrated in Figures 3 and 4. A comparison of the spectral densities of these plates with those used in the earlier work seems to indicate that the recent plates were less sensitive in the green. Nevertheless the spectral energy distribution found for the light of the firefly is in excellent agreement with that previously observed; and presumably the greater variation in spectral photographic sensitivity has not appreciably affected the accuracy of the determination of the spectral energy distribution of the luminescent fungus, which has its maximum light emission in the green, where the plate is the least sensitive.

For details in reducing the data the reader is referred to previous publications. It will be sufficient to add that for conrenience the 2 -seconds spectral density curve of the glowlamp was taken as

${ }^{5}$ Ives and Coblentz, B. S. Bull. (No. 132), 6, p. 321; 1910. Coblentz, A Physical Study of the Firefly, Publ. No. 164, Carnegle Inst. of Washington; 1912.

- Coblentz, Publ. No. 164, Carnegie Inst. of Washington; 1912. 
Scientific Papers of the Bureau of Standards, Vol. 21

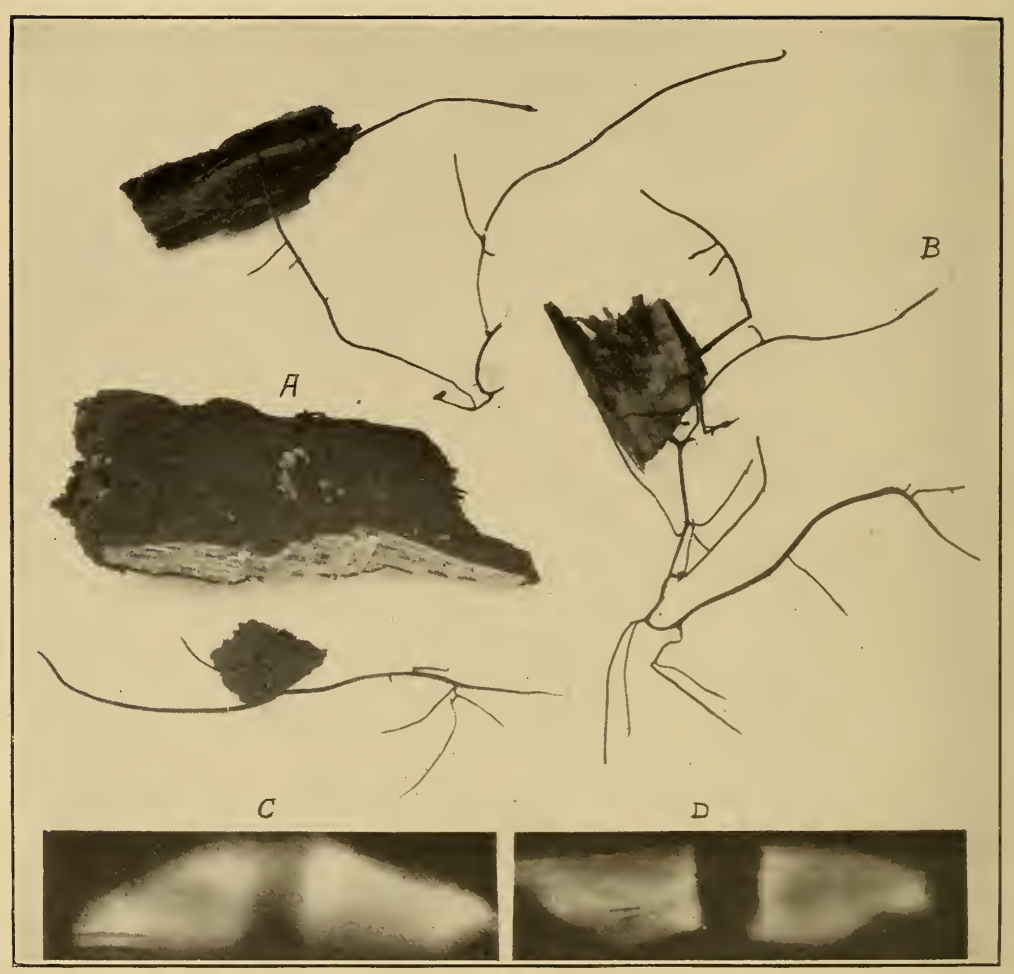

FIG. 1.-Luminous wood 
a unit of comparison. Hence the 10-seconds density curve represents 5 units, etc. At the wave lengths where the density curves of the luminous wood intersect the spectral density curves of the glowlamp it is assumed that the plate has been acted on by the same amount of energy. For example, at the intersection of the 20 -seconds density curve of the glowlamp with the curve for the luminous wood the "ratio of densities" is 10 . Multiplying this value by the relative spectral energy distribution of the glowlamp at the wave lengths gives us the spectral energy of the luminous wood at these wave lengths.

In spite of the apparent irregularity in the spectral density curves of the different times of exposure, after multiplying the "ratios of densities" by the spectral energy in the glowlamp at the various wave lengths, the results give a smooth curve. From this it appears that the method is fairly reliable for determining the spectral energy distribution of faint sources of radiation.

\section{OBSERVATIONS ON LUMINOUS WOOD}

From the very beginning of investigations in bioluminescence it was found that water, oxygen, and a photogenic substance are necessary for the production of light. The fungus (Agaricus melleus) seems to be no exception to this rule.

Pieces of decaying oak wood were kept in an ordinary glass fruit jar, which is provided with a glass lid and a metal locking device, rendering it air-tight. The luminescent material was under observation for over a year. In the meantime the rood was kept moist by occasionally filling the jar with water to wash out what at first was thought to be the brown stain of tannic acid in the oak wood, but which, from information gained by subsequent reading on the subject and by direct observation, was probably owing, in part, to a brownish liquid which is exuded by small, hairy, grayish-colored tufts (1 to $1.5 \mathrm{~mm}$ high) which appeared on the surface of the wood. The tops of these tufts (fig. 1, $A$ ) contained a minute drop of brownish colored liquid which wets these hairy protrusions when stroked with a needle.

In the evening, after the eye had become dark adapted, the glass cover was removed. It was found that when the jar was tightly covered for some hours the luminescence ceased, but the moment the lid was removed the uppermost pieces of wood began to glow. This glowing slowly extended to the bottommost samples. During the night the whole container was aglow with the luminescent wood. After a few weeks the outer surfaces of the pieces of wood became covered with fine strands of a brownish-colored growth of mycelium, which did not glow, and by the end of 6 weeks the outer surfaces 
ceased to glow on opening the jar. However, on splitting these pieces of wood the freshly exposed surfaces glowed brightly. In fact, some of the most luminous" material used in photographing the spectrum were obtained by splitting some of the most brittle samples which had been in the glass jar for about 10 weeks.

While the presence of oxygen seems to be of some importance in the active metabolism of this fungus, it seems to be needed in only small quantities. For example, placing samples of dimly and of brightly luminescent wood in a flask of pure oxygen did not seem to increase the brightness, although the luminescence continued for more than a day.

In the present investigation of the luminous organism (Agaricus melleus) in decaying wood, in which the intrinsic brightness is much lower even than the faint glow emitted by a firefly, it was found that the apparent color of the light emitted depends to a great extent upon the adaptation of the eye. The material used in photographing the spectrum consisted of a bucket full of chips, splinters, and pieces of roots cut from the underground part of an old oak stump. On first entering the darkened spectrometer room, during the daytime, it was found that the samples which glowed the brightest had a greenish-yellow tint as compared with the faintly glowing material, which appeared more bluish-white. After the lapse of half an hour, when the eye had become dark adapted, this greenish-yellow color disappeared, and there remained only a difference in brightness among the samples.

Hence, in selecting the brightest samples for photographing the spectral light, advantage was taken of this color phenomenon by picking out the most brilliant greenish-yellow colored samples on first entering the spectrometer room.

Probably there is a real difference in the color of the light emitted by various fungi, as described by various observers. But it is erident that mere naked-eye observations are not sufficient to give an accurate description of the composition of the light emitted.

It will be shown below that the light emitted by this fungus (Agaricus melleus) is composed of wave lengths extending over a greater range of the visible spectrum than obtains in the firefly. Hence a difference in the color of the light emitted by these two objects is to be expected.

As observed by others, the mycelium is very easily injured even by accidently touching the luminescent surface while photographing the spectrum. As already stated the light emitted is controlled by the presence of oxygen and by the time of life and development of the plant. In Figures $1 \mathrm{C}$ and $\mathrm{D}$ is shown a photograph made by placing the surface of the luminescent wood in contact with the photographic 
plate. The central dark region shows the path of the steel chisel used in splitting the wood.

When the wood was first rent asunder the surface was nonluminous. The photograph was made five hours later when the surface was at its maximum brightness. At that time the part touched by the chisel was slightly luminous, but not sufficiently so to affect the photographic plate on short exposures.

Contact photographic exposures of 5 to 60 seconds were made without overexposing the plate. This is owing partly to the low intensity of the light emitted, and also owing to the fact that the maximum emission lies in the green where the photographic plate has a low sensitivity.

This picture shows, as found by others, that the most intense light emission is obtained from the broken and torn mycelium on the surfaces that had not been touched in splitting the wood. An interesting observation is that the brightest glow occurs on that portion of the surface close to the part compressed by the chisel. Whether this is owing to the greater number of mycelia compressed into this space or to stimulation remains undetermined.

Soon after beginning the work it was observed that the maximum light emission occurred in 3 to 5 hours after splitting the wood, after which it continued to be very luminous for 5 to 8 hours. Advantage was, therefore, taken of this information in order to provide a continuous supply of brightly glowing material for producing a spectrum. Samples of brittle wood which were 2 to $3 \mathrm{~mm}$ in thickness and glowing on both sides seemed to be the brightest. Whether this is owing to the presence of light transmitted through the wood, or to the penetration of oxygen into, and to the luminescence of the mycelium within, the wood remains to be determined.

\section{EFFECT OF SUNLIGHT AND ULTRA-VIOLET LIGHT}

An examination was made of the effect of sunlight upon the luminescent fungus. The observer remained in the dark room, while an assistant exposed part of the luminous wood to the sun for, say, 10 to 20 minutes. During this time there was but little drying of the wood (which kills the mycelium) and no difference was observed in the brightness of the exposed and the unexposed portions of the luminous surface. Similarly, exposing the glowing fungus to the radiation from a quartz-mercury arc lamp had no effect upon the brightness of the luminescence.

\section{OBSERVATIONS ON THE ISOLATED MYCELIUM}

As found in the decaying wood the luminous mycelium is indistinguishable from the rest of the background. After the material had been kept in the glass jars for some weeks glossy, rootlike, red- 
dish-brown tubular filaments, consisting of a thin, loose, outer integument over a strong fibrous, whitish-colored central thread, were observed growing from the pieces of wood. A characteristic of this growth is that the filaments project out normal to the surface. The lips of these filaments, over a length of 2 to $3 \mathrm{~mm}$, are translucent, greenish-white or yellowish-white in appearance, as shown in Figure $1, B$, and emit a very faint light. These filaments increased in length (10 cm or more) and branched into a complex mass, adhering to other pieces of wood when coming into contact with them. The older deep-brown parts ceased to emit light; but the very light-colored parts and the translucent terminals continued to be luminescent after a year's observation. The white fibrous interior did not seem to emit light. Some of these pieces of wood were placed in ground under a large bell jar to retain the moisture, hoping to grow the top part of the fungus which is luminous, but at this writing the attempt has been unsuccessful. A great many filaments grew out of the wood, through the ground, and out from under the bell jar.

\section{THE SPECTRAL ENERGY CURVE OF THE LUMINOUS FUNGUS}

In Figure 3 is shown the photographic spectral-opacity curves produced by the mycelium of the luminous fungus, Agaricus melleus, and also of the standard glowlamp. From the intersections of these two sets of curves and the spectral energy distribution of the standard glowlamp the spectral energy distribution of the luminous fungus is obtained. The two density curves are for 24 and 71 hours' exposure.

As shown in Figure 5 the energy emitted is distributed over a wider region of the spectrum than has yet been observed in any other luminous organism or phosphorescent material; extending from $0.47 \mu$ in the blue to $0.68 \mu$ in the orange red. The maximum emission, as shown in Figure 2, is at $0.52 \mu$ in the green, producing a greater darkening of the photographic plate than obtains in the photograph of the spectrum of the glowlamp. The maxima of emission of the 71hour exposure, as worked out from two sets of exposures of the standard glowlamp, are in good agreement.

The spectral energy distribution is quite unsymmetrical. While this may be owing to difficulties in the proper evaluation of the characteristic of the photographic plate, this unsymmetrical distribution may be owing to a small maximum emission in the region of $0.55 \mu$. There are no data at hand to refute such a possibility. In the fluorescence and phosphorescence phenomena investigated by Nichols, which extend over a much narrower region of the spectrum, it was observed that the light emitted is composed of one or more series of overlapping components. Whether the light is emitted by minerals,

7 Nichols, Proc. Amer. Philosoph. Soc., 55, p. 496, 1916; 56, p. 258, 1917. 
excited to phosphorescence by an extraneous radiation, or whether it is emitted by animal or plant organisms, by processes which are as yet undiscovered, there is nothing in theory or experiment to indicate what one should expect to find in the case of a spectral energy distribution extending over the greater part of what constitutes the visible spectrum, and producing the faintly tinged, greenish-white light observed in the luminous fungus. While this color may be owing partly to the low luminosity and the state of adaptation of the eye, as already mentioned, the experimental data presented show that the whitish color of the light is owing to the wide spectral range of the radiation

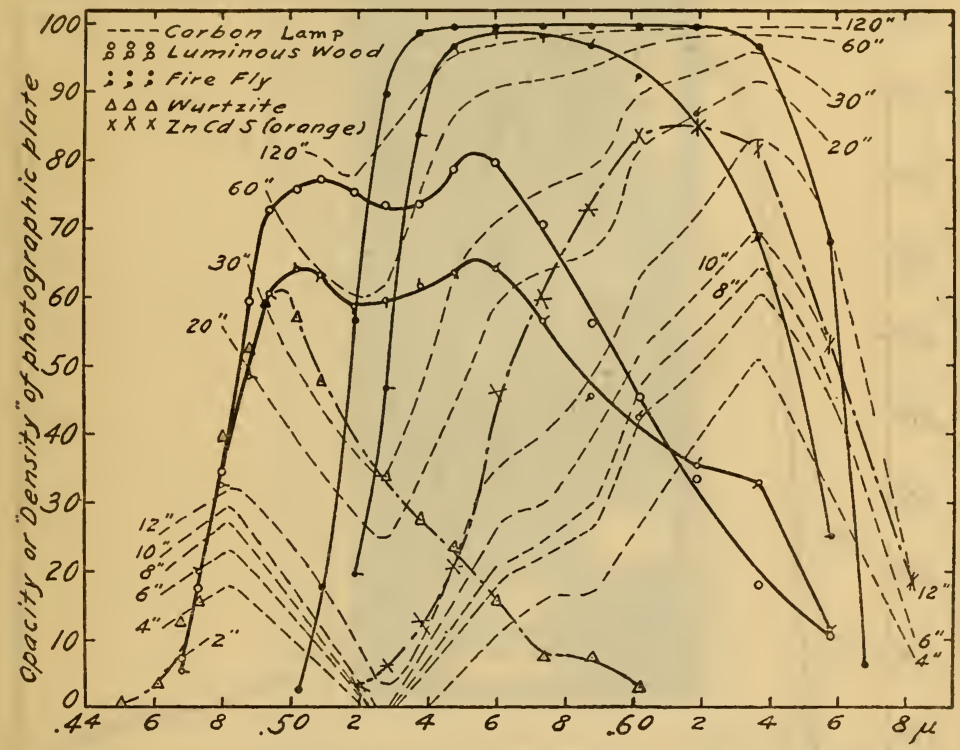

FIG. 3.-Spectrum density curves for different exposures in seconds

emitted, extending from 0.43 to $0.68 \mu$, as viewed on the photographic plate, and from 0.47 to $0.66 \mu$ as recorded by spectral energy measurements.

\section{SPECTRAL ENERGY DISTRIBUTION OF THE LIGHT EMITTED BY CYPRIDINA}

As already mentioned, the dried pulverized crustacean, Cypridina, was used. On moistening the material there is a bright flash of bluish-green light. Some difficulty was experienced in presenting the luminous material before the spectrometer slit. The best procedure was to place the dry powder upon a block of hardwood, sawed across the grain, moisten it with water, and rub the material with a splinter of wood while holding it tilted at an angle of $45^{\circ}$ in front 
of the spectrometer slit. As the dried material was further pulverized and moistened very bright flashes were obtained. By proceeding in this manner for 1,2 , and $31 / 2$ hours good photographs were obtained of the spectrum, as shown in Figure 2.

The density curves are shown in Figure 4. From these curves and from the spectral energy distribution of the glowlamp were obtained the two spectral energy curves of the luminescent crustacean, shown in Figure 6.

The spectral energy distribution is symmetrical, extending from 0.41 to about $0.62 \mu$ with a maximum at $0.48 \mu$ in the blue green.

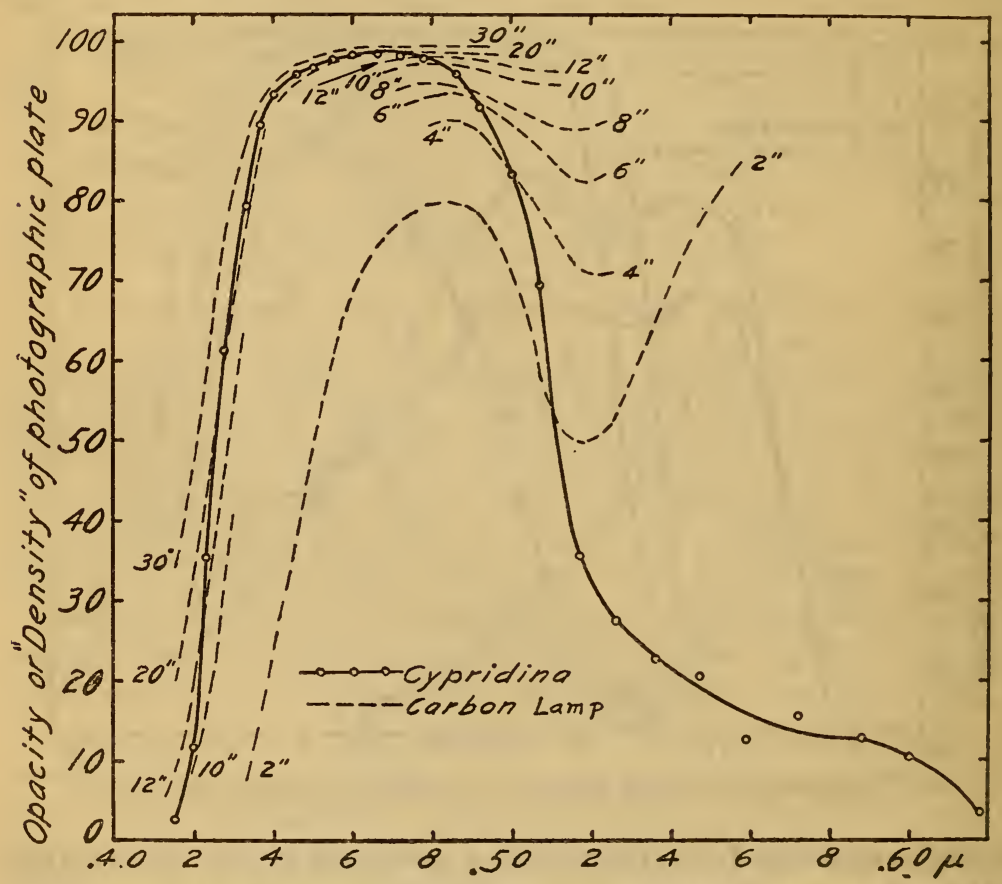

FIg. 4.-Plate density curves for Cypridina and the light of the carbon lamp

As shown in Figure 4, the light emission in the orange-red part of the spectrum is extremely weak as compared with the maximum.

The light emission of the living organisms in the ocean, as viewed from a large boat does not seem quite so deep blue green. But this may be owing to the fact that they are seen at a distance as small clumps or colonies in the turbulent water.

\section{SPECTRAL ENERGY DISTRIBUTION OF THE LIGHT OF THE FIREFLY}

As a check on the other work an examination was made of the spectral energy distribution of the light emitted by the firefly, 
Scientific Papers of the Bureau of Standards, Vol. 21

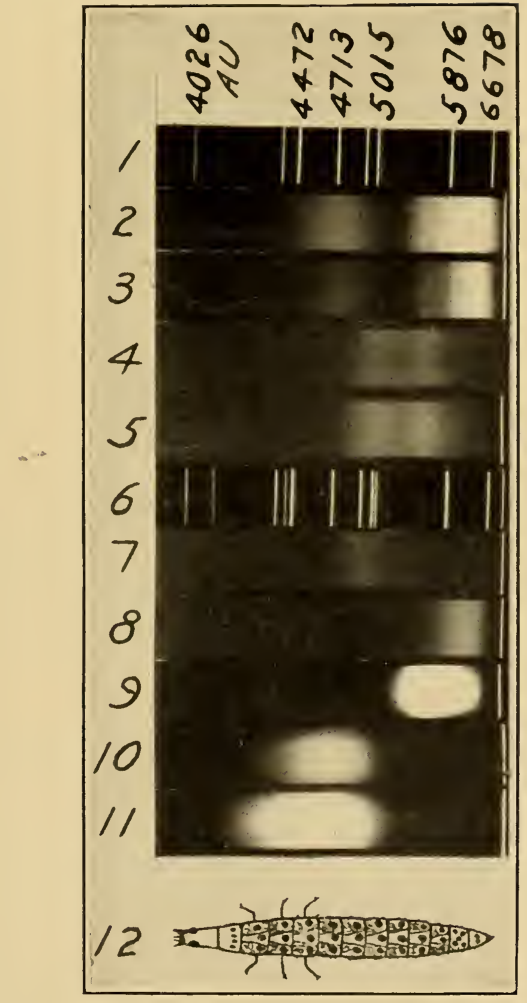

FIG. 2.-Spectrograms

1 and 6, Helium; 2 and 3, carbon glowlamp; 4 and 5, luminous wood, 24 and 71 hours; 7 , wurtzite, $\mathrm{ZnS}$; 8, orange-cclored $\mathrm{ZnCd}$; 9 , firefly; 10 and 11, crustacean (Cypridina), 1 and 3 hours; 12 , Sumatra glowworm 
Photinus pyralis. The season was already late (August 21, 1925) and only two specimens (males) could be found. The first one flashed a number of times and then emitted the glow characteristic of fireflies that are exhausted. By lightly stroking the luminous segments with a wooden toothpick this (bright) glow was prolongred for an hour. The second specimen could be stimulated to glow brightly (but not to flash) for an hour.

In both spectrograms (one of which is shown in fig. 2) the plate is overexposed in the center of the bright emission band, although

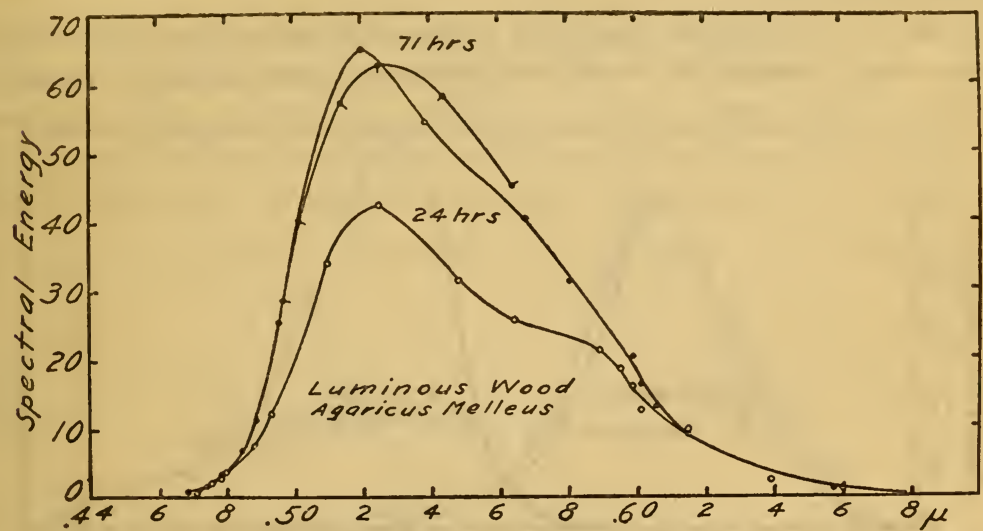

Fra. 5.-Special energy distribution of light emitted by a fungus, Agarious melleus

the spectrometer slit was open only $0.5 \mathrm{~mm}$. Nevertheless, from the density curves illustrated in Figure 3 , good spectral energy curves were obtained, especially at the sides of the maximum. They are in excellent agreement with previous measurements in which the photographic plates were fresher and had a more uniform spectral photographic sensitivity.

As shown in Figure 6, the spectral energy distribution of the light emitted by Photinus pyralis is unsymmetrical, extending from 0.50 to $0.68 \mu$ with a maximum at $0.56 \mu$.

\section{COMMENTS ON FIREFLIES AND GLOWWORMS IN SUMATRA}

One of the writers had an excellent opportunity to study the fireflies and glowworms in Benkoelen, Sumatra. One glowworm $(3 \mathrm{~cm}$ in length) was kept in an open tin box for a month. It usually remained curled up under a clump of growing sod, but at night it would emerge for food and mating as is the habit of this animal.

As shown in Figure 2, this glowworm has three lines of luminous organs, with a double light in the central line in the first segment and a set of 5 lights in the next to the last segment. It has a total 
of 38 luminous organs, emitting a brilliant greenish-blue light which is very conspicuous as observed along a path or roadside at dusk.

The fireflies are small, being 6 to $8 \mathrm{~mm}$ in length, and in that respect they resemble our Photinus scintillans. But in their flashing they are entirely different from the latter which emit two short flashes, then remain quiet for a while. The Sumatra fireflies stand on blades of grass, or fly about, flashing sometimes at the rate of 70 or. 80 times per minute. The volume of the light seems greater than that of our Photinus pyratis and the color is perhaps a little more yellowish.

Most of the fireflies caught at dusk were males; but after dark, when many would be found on blades of grass flashing rapidly,

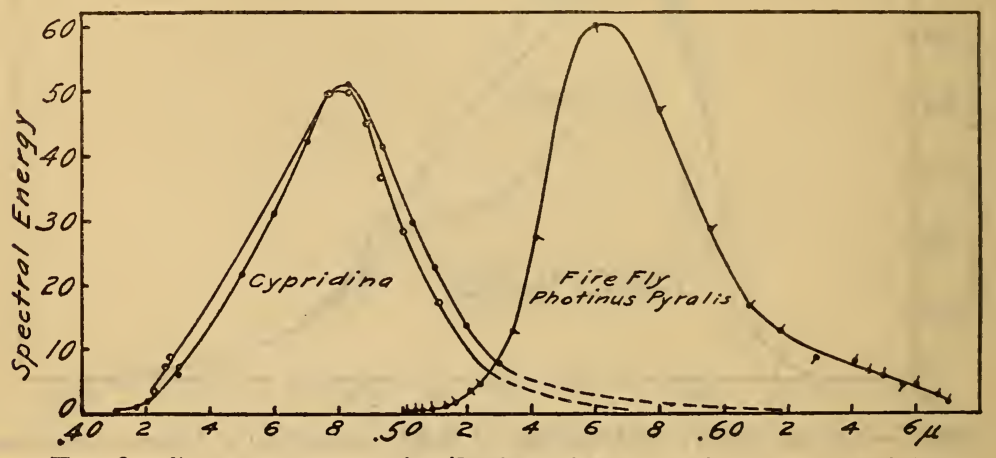

FrG. 6.-Spectral energy distribution of light emitted by Cypridina

about 75 per cent of the total number caught were females. The larvæ of these fireflies are small and emit a greenish light, similar to that of our native species.

\section{SPECTRAL ENERGY DISTRIBUTION OF SOME PHOS- PHORESCENT ZINC SULPHIDES}

It was of interest to compare the spectral energy distribution of the light emitted by phosphorescent substances with that of animal and plant organisms in order to show the very limited spectral range of wave lengths emitted. This could be done very conveniently in view of the fact that just a little while previously Dr. A. A. Guntz ${ }^{8}$ had sent us his thesis on a study of phosphorescent zinc sulphides as well as several samples of the materials investigated.

Two samples of phosphorescent sulphides painted on white cardboard were examined: Wurtzite, $\mathrm{ZnS}$, emitting a greenish-blue light, and a buff-colored material, $\mathrm{ZnCdS}$, which emitted a rich orangecolored phosphorescent light.

\footnotetext{
${ }^{8}$ Guntz, Thesis Series No. 1012 , order No. 1848 , presented to the faculty of Sciences of the University of Paris; 1925.
} 
These materials were excited to phosphorescence by exposing them to the light of a small tungsten flash-light lamp. The card was then placed in front of the spectroscope slit for a short time, after which the material was again exposed to the light of the flash lamp. By proceeding in this manner for 40 minutets good spectrograms (shown in fig. 2) were obtained of both materials.

The spectral energy curve (fig. 7) of the phosphorescent light emitted by wurtzite, $\mathrm{ZnS}$, is quite symmetrical, extending from 0.46 to $0.61 \mu$, with a maximum at $0.52 \mu$ in the blue green.

The spectral energy distribution (fig. 7) of the $\mathrm{ZnCdS}$, emitting an orange-colored phosphorescent light, extends from $0.5 \mu$ in the green to wave lengths greater than $0.7 \mu$. It seems to have two maxima; one at $0.52 \mu$, coinciding closely with that of $\mathrm{ZnS}$, and a more intense maximum at $0.61 \mu$ in the orange-colored part of the spectrum. As already mentioned, there seems to be nothing

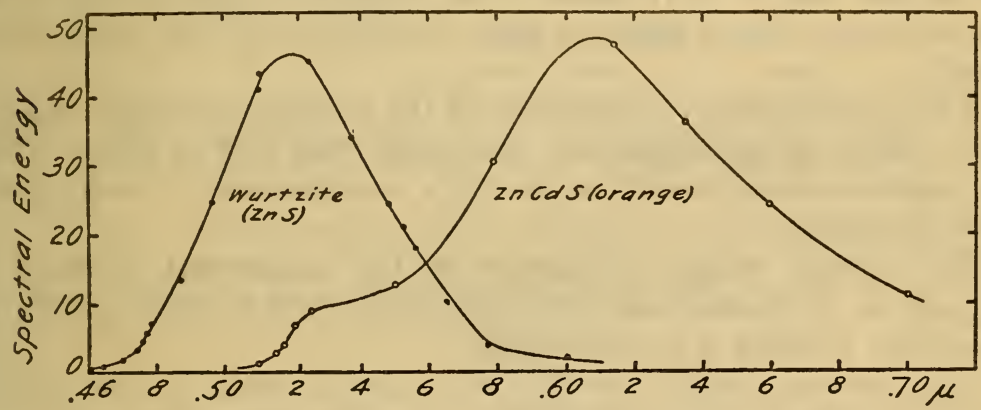

FTG. 7.-Spectral energy of phosphorescent substances

novel in observing a phosphorescence band having overlapping maxima.

The results as a whole are interesting in showing the limited range of the spectral energy distribution of this type of radiation as compared with a purely thermal radiation in which the partition of energy extends over a much wider range of the spectrum.

\section{SUMMARY}

One of the foremost problems now occupying the attention of investigators is the production of light and the improvement of our methods of illumination. All our methods of light production are accompanied by a large amount of thermal radiation which can not be utilized in vision. Hence, the interest in the spectral energy distribution in phosphorescence found in some inanimate substances and in the bioluminescence found in some plants and animals in which the radiation emitted is comprised with a very narrow region of the visible spectrum. 
The present investigation is a further contribution to this subject. The paper deals with the spectral energy distribution of the light emitted by a luminescent fungus (Agaricus melleus) found in decaying wood which emits a faintly tinged greenish-white light, popularly konwn as "box fire," and also a luminescent crustacean (Cypridina) which emits a bluish light. For comparison further data are given on the spectral energy distribution of the light of a firefly (Photinus pyralis). Data are also given on the spectral energy distribution in the spectra of several phosphorescent sulphides of zinc.

The spectral energy distribution of the luminescent light was obtained by photography upon a panchromatic plate. For this purpose spectrum density curves of the luminescent material were compared with similar curves of a standard glowlamp of known spectral energy distribution. This required an exposure of an hour for the crustacean and 30 to 71 hours, with frequent renewal of the fresh material, in order to obtain a good spectrogram of the luminescent wood.

The spectral energy distribution of the luminescent fungus Agaricus melleus is unsymmetrical, extending from 0.43 to $0.67 \mu$ with an intense emission maximum at $0.52 \mu$, and probably a weak maximum at $0.58 \mu$.

The spectral energy distribution of the luminescent crustacean, Cypridina, is symmetrical, extending from 0.41 to $0.62 \mu$, with a maximum at $0.48 \mu$ in the blue green.

The spectral energy curve of the light emitted by the firefly, Photinus pyralis, is unsymmetrical, extending from 0.50 to $0.68 \mu$, with a maximum in the region of $0.565 \mu$, verifying previous observations.

The spectral energy distribution of wurtzite $\mathrm{ZnS}$ extends from 0.46 to $0.61 \mu$, with a maximum of $0.52 \mu$ in the green-blue part of the spectrum.

The orange-colored sulphide of $\mathrm{ZnCdS}$ seems to have two emission maxima; one at $0.52 \mu$ in close coincidence with that of $\mathrm{ZnS}$, and a more intense maximum at $0.61 \mu$, which produces the dominant color, orange, of the phosphorescent light.

Washington, July 15, 1926. 